\title{
Innovation Ultrasonic Assistant Soldering in Electronics
}

\author{
Vladimir Lanin* \\ Department of Electronic Technique and Technology, University of Informatics and Radioelectronics, Minsk, Belarus \\ *Corresponding author: Vladimir L. Lanin, vlanin@bsuir.by
}

\begin{abstract}
Innovative approaches in ultrasonic assistant soldering consists of increasing the activity of cavitation and accelerating diffusion processes at the interface between the solder and the soldering material. Besides that, it improves the effectiveness of cavitation processes in melts by saturating gas cavities with diameters that are smaller than the resonant sizes of cavitation germs. Gas saturation of liquids and melts raises level of cavitation pressure by 20-25\%, that intensifies US processing of cleaning, soldering and metallization. Modelling diffusion process showed that the US activation increased the concentration of diffusing elements of $\mathrm{Zn}$ and $\mathrm{Al}$ in the interface depth by 15-20\% on average, and the combined activation by the US and electric field increased it by $30-45 \%$. Furthermore, as the energy quantity adsorbed by melt increases, increased amplitude and frequency of US vibrations induces concentration rise. The heat energy was also boosted by combining the activation of the melt-soldered material system with US vibrations energy and high current pulses. This allows for a faster increase in soldering temperature, as well as improved solder wettability.
\end{abstract}

Keywords: Ultrasonic; Cavitation; Gas cavities; Electric field; Melts

Publication date: May 2021; Online publication: May 31, 2021

\section{Introduction}

The effects of intense ultrasound on liquid media include acoustic cavitation, which causes pulsations and the collapse of cavitation bubbles; ultrasonic capillary effect; sonoluminescence; vortex effects in the form of micro and macro flows; and acceleration of diffusion processes, among others ${ }^{[1]}$. The greatest number of secondary physical effects is caused by cavitation, according to an analysis of intense ultrasonic impacts in liquid media (Figure 1.). Due to conditions created for the formation of chemical bonds between oxides at the interface, local thermal effects arising from the collapse of cavitation bubbles are used in ultrasound metallization of nonmetallic materials such as ceramics, glass ceramics, ferrites, etc. ${ }^{[2]}$ Dynamic effects ensure the removal of grease and oxide films from the surfaces of materials, which is the physical basis for ultrasonic cleaning in liquid media ${ }^{[3]}$ and ultrasonic brazing of hard-to-solder materials ${ }^{[4]}$. Some primary effects, such as the electrokinetic, wetting, have not yet been widely used in technology due to the limited possibilities of implementation.

It is critical to produce a cavitation area with adjustable erosive activity when ultrasonic (US) processing in liquids and melts is used for pollution removal, metallization of non-metallic materials, and scatter micro- and nano-dimensional powder materials. However, nonuniformity of distribution US pressure and differences in intensity cavitation define US fields in baths with varied numbers of radiators [5].

In liquids the quantity of cavitation germs does not give in to the exact account, as it depends on many factors such as, gas maintenances in a liquid, quantities of firm particles, ambient temperatures, external pressure, etc. Not all cavitation germs participate in cavitation process, but only those from them which 
under action US pressure have reached of the critical sizes. The cavitation threshold decreases, if the liquid environment to influence an ionizing radiation or an electric current. Both of these influences are connected with formation of new cavitation germs in the liquids. However, at these methods of activation cavitation process remains non-stationary and difficultly operated ${ }^{[6]}$.

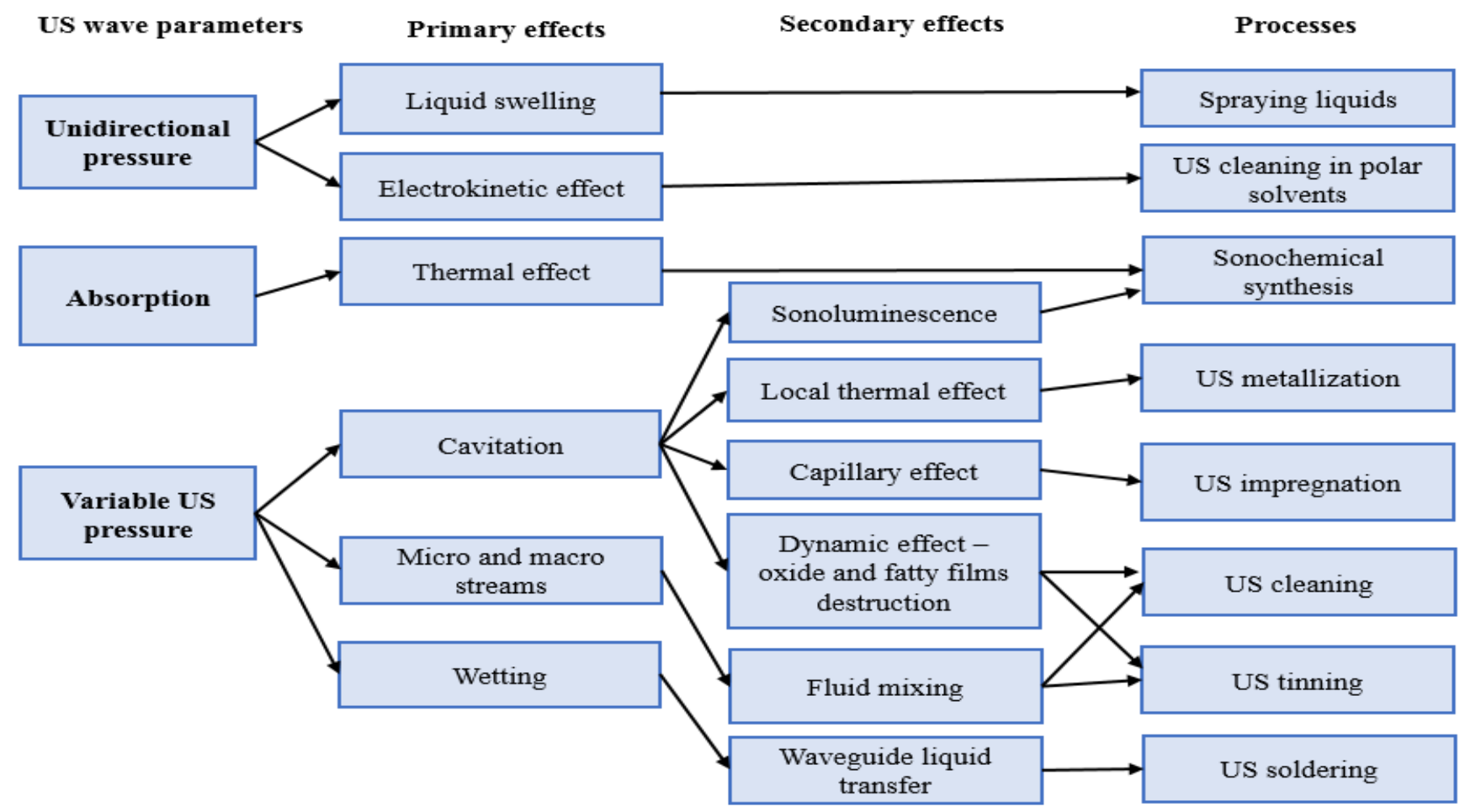

Figure 1. Ultrasound effects in liquid media and their application

Innovative approaches in ultrasonic assistant soldering, consist of increasing the activity of cavitation and accelerating diffusion processes at the interface between the solder and the soldering material. Perspective direction in increase of cavitation processes efficiency is gas saturation of a liquid by cavities, with the sizes not exceeding the resonant sizes of germs, i.e. (10-50) $10^{-6} \mathrm{~m}$. Increase in the sizes of cavities will lead their premature collapse, and very small sizes complicate cavitation growth of cavities in US field. Another direction is the introduction into the solder melt of insoluble particles of diffusion-active metals, on which nuclei of cavitation bubbles can form.

The replacement of traditional solders with lead-free alloys which has caused a number of problems in the field of solder metal science. Eutectic alloys of the $\mathrm{Sn} \mathrm{Ag-Cu} \mathrm{(SAC)} \mathrm{type} \mathrm{are} \mathrm{widely} \mathrm{used}$ despite the higher cost (3 times higher than cost of solders) due to ease of use and low creep ${ }^{\text {[7] }}$. However, the reliability and mechanical properties of soldered joints very much depend on microstructure of solder, the morphology, and thickness of intermetallic compounds at the interface. The formation of $\mathrm{Ag}_{3} \mathrm{Sn}$ and $\mathrm{Cu}_{6} \mathrm{Sn}_{5}$ intermetallides has main negative effect on fatigue processes and causes cracking at solder - metal interface ${ }^{[8]}$.

In some cases, to obtain high-strength aluminum butt joints with corrosion resistance, ultrasonic soldering was conducted using quasi-melting Sn-Zn hypereutectic alloy. Ultrasonic vibrations were applied at soldering temperatures ranging $220-300^{\circ} \mathrm{C}$ through $\mathrm{Al}$ rods without a solder bath ${ }^{[9]}$. To improve the wettability of ceramic material with $\mathrm{Zn}-\mathrm{Al}-\mathrm{Mg}$ solder, soldering with the assistance of active ultrasound was employed. The tensile strength of $\mathrm{Zn}-\mathrm{Al}-\mathrm{Mg}$ soldering alloys ranges from 82 to $169 \mathrm{MPa}$; the highest 
strength was observed in the solder with the lowest $\mathrm{Mg}$ content ${ }^{[10]}$.

Ultrasonic energy activates the molten solder alloy and causes different structure of soldering joints. Analysis of sample micro sections with copper soldering pads confirm the influence of ultrasound on the structure of solder joints. The highest share strength, have been achieved for solder joints which were prepared by ultrasonic soldering on pads with copper surface finish. It is possible to use this technology for creating the electrode system for solar cells ${ }^{[11]}$.

To modify lead-free solders used to form contacts in electronics, alloying adhesive active additives in the form of antioxidants, such as $\mathrm{Ce}, \mathrm{Ti}$, as well as metals that reduce the growth of intermetallic compounds like Ni, Ge, are most promising. So, in BALVER ZINN solder, due to introduction of number of metal additives like $\mathrm{Cu}-0.6-0.7, \mathrm{Ag}-0.05, \mathrm{Ge}-0.005-0.007, \mathrm{Ni}-0.04-0.06^{[12]}$, the mechanical strength is significantly improved, number of inclusions and solder bridges are reduced.

The use of ultrasonic vibrations during modification of solder allows to solve these problems. The main effects of action of cavitation and micro-streams on liquid metals are acceleration of nuclei primary formation, and the initiation of secondary nucleation of crystallization centers due to physical mixing and acceleration of diffusion. Ultrasonic modification of alloys is characterized by such effects as grain refinement, improved structure uniformity, wetting, and mechanical properties of soldered joints ${ }^{[13]}$. However, to obtain the best properties of soldered joints with lead-free solders in electronics, optimization of parameters of ultrasonic effect on solder melts is necessary.

Interest in flux-free US soldering processes is caused by transition to lead-free solders and environmental problems of soldering in electronics. To form high-quality compounds, methods and devices for local ultrasonic activation of solder melts are used. US soldering is preferable for electronic applications because flux is a corrosive agent and it is necessary to remove flux resides as to ensure adequate service reliability of electronic assemblies ${ }^{[14]}$.

A new approach in melt processing technology involves using a high frequency electromagnetic induction coil to induce ultrasonic pressure waves that cause cavitation in alloy melts. This presents an alternative "contactless" approach to conventional US immersed probe techniques. The method can potentially offer the same benefits of traditional ultrasonic treatment (UST) such as degassing, microstructure refinement and dispersion of particles, but avoids melt contamination due to probe erosion prevalent in immersed sonotrodes. An added benefit is that the induction heating and stirring produced by Lorentz force, enables a larger melt treatment volume ${ }^{[15]}$.

The main objective of this work is to develop methods to improve the efficiency of ultrasonic soldering processes for the mounting of modern electronic devices.

\section{Fundamentals}

For estimation intensity cavitation processes in liquids use the index of a cavitation defined by the relation of total volume of cavities $\Sigma V_{\kappa}$ to volume of a liquid $V^{[16]}$ :

$$
\chi=\Sigma \mathrm{V}_{\mathrm{K}} / \mathrm{V}
$$

The total volume cavities $\Sigma V_{\kappa}$ is equal:

$$
\sum V_{\kappa}=4 / 3 \pi \cdot N \cdot R_{\max }^{3},
$$

where $N$ - number cavities, $R_{\max }$ - radius of cavities in a stage of the greatest expansion. 
Experimental data testify, that cavitation area is, as a rule, in a near zone US radiator, therefore US activated liquids volume it is possible to present as product of the area of radiator $S_{r}$ on height of cavitation area $h$. Taking into account these assumptions expression (1) will assume the following kind:

$$
\chi=\frac{4 \pi \cdot N \cdot R_{\max }{ }^{3}}{3 S_{r} h} .
$$

Using the equation of ideal gas condition receive:

$$
p V=m_{M} N R T
$$

where $p$-gas pressure, $V$-gas volume, $m_{M}$ - weight of gas molecules, $R$-gas constant, $T$-gas temperature.

Regulating pressure and speed of gas distribution it is possible to operate number of the gas molecules $N$ entered into a near zone of US radiator as potential cavitation germs.

At gas activation to liquid, from the compressor with the help of nozzle submitted air or inert gas argon with pressure to $0,1-0,2 \mathrm{MPa}$. For uniformity of the size of gas cavities in a liquid on exit of nozzle is established the filter-membrane with cells which were formed by laser radiation with high accuracy and repeatability of the sizes. The nozzle includes two porous filters with the sizes of apertures $2-5$ microns. For creation of cavities of the micron sizes it is necessary to satisfy following conditions: pressure of air should not exceed 0,2-0,3 MPa, and the distance between apertures in the filter in comparison with diameter of cavities should be considerable. These conditions in certain degree prevent association of cavities of the micron sizes in the big gas cavities.

The resonant frequency of cavitation cavities collapse depends both on its radius $R p$, the surface tension of the melt $\sigma$ and on density $\rho$ according to Minert's expression:

$$
f_{p}=\frac{1}{2 \pi R_{p}} \sqrt{\frac{3 \gamma}{\rho}\left(P_{o}+\frac{2 \sigma}{R_{p}}\right)}
$$

where $\gamma$-isothermal coefficient, $P o-$ external pressure.

The action of the US field energy on the melt increases the diffusion coefficient and activates the nucleation process ${ }^{[17]}$

$$
D^{\prime}=D_{0} e^{-\frac{E-\Delta E}{R T}}
$$

where $D_{0}$ is the pre-exponential factor, $E$ is the energy of the diffusion activation, $\Delta E$ is the change in the energy of the diffusion activation in the US field, and $R$ is the gas constant.

If the US energy introduced into the melt without taking into account losses activates the diffusion process, then

$$
\triangle E=0.5 \rho c(A \cdot \omega)^{2},
$$

where $\rho c$ is acoustic impedance, $A$ - amplitude of fluctuations, $\omega-$

circular frequency. 
In the US field, the force $F$ acts on the diffusing particles, and, under its action, the substance particles move with the average velocity overcoming the force of medium friction $F_{f}$

$$
V=v F-\frac{F_{f} d}{\eta S_{p}}
$$

where $\mathrm{v}$ is the particle mobility, $d$ - medium layer thickness, $\eta$-dynamic viscosity, $S_{\mathrm{p}}$ - particle area. The strength of the ultrasonic field, affecting the particle flow section S:

$$
F=\rho \cdot \omega A \cdot S
$$

At the activation in the US field, a flux of particles, which move under the action of the force of US vibrations $F$, is added to the diffusion flux; then, the entire flux amounts to ${ }^{[18]}$

$$
J=-D^{\prime} \frac{\partial C}{\partial x}+V C_{1} \cos \alpha=-D^{\prime} \frac{\partial C}{\partial x}+\left(v \rho c \omega A S-\frac{F_{f} d}{\eta S_{p}}\right) C_{p} \cos \alpha
$$

where $C_{\mathrm{p}}$ is the concentration of mobile particles, and $\alpha \beta$ is the angle between the vector of the force of the US field and the diffusion flux vector. It follows from Equation (10) that activation by the US field energy leads to an increase in the diffusion flux.

\section{Experimental}

At gas activation of melt submitted on the converter, 1 electric fluctuation by frequency $20-44 \mathrm{kHz}$ and amplitude 50-75 mkm from US generator. In a solder melt immersed US radiator 2 so that a backlash between it and soldered element 5 made 0,25-1,5 $\mathrm{mm}$. Through tube 3 and capillary-porous body 4 in a direction to a soldered surface in a melt of solder 6 entered inert gas - argon (Figure 2.). The sizes of capillaries in a porous body are chosen such that the sizes of formed gas cavities did not exceed the resonant sizes of cavitation germs in a melt of $\mathrm{Sn}-\mathrm{Pb}$ solder, example: $10-50$ microns ${ }^{[19]}$.

Quantification the spectral density of cavitation noise it is possible to determine the cavitation intensity in liquid surrounding ${ }^{[20]}$. The greatest spectral density of cavitation noise is in the frequency band from $10^{\text {th }}$ up to $40^{\text {th }}$ harmonics of the base frequency of the ultrasonic converter at acoustic pressure $2 \cdot 10^{5} \mathrm{~Pa}$ (Figure 3.) ${ }^{[21]}$. It is offered to quantification cavitation pressure in liquid media by measure the square of a noise level over the range of the greatest spectral density of a cavitation noise The scheme of a cavitometer (Figure 4.) consists of a piezoelectric sensor, an active filter creating a shear sternness of frequency characteristic curve less than $24 \mathrm{~dB}$ an octave, a square-law detector, and a recording device. The indications of this device have linear dependence on active power in range of $0-2 \mathrm{~kW}$. The cavitometer measures cavitation pressure from 5 up to $5 \cdot 10^{4} \mathrm{~Pa}$ in the frequency band $18-60 \mathrm{kHz}$ with accuracy $\pm 10 \%$.

The pressure in cavitation area is perceived by measuring probe 1 , attached to a piezoelectric transducer 3 with the elastic waveguide 2 . The electrical signal from the transducer 3 comes to the amplifier 4 , arranged in a body of the sensor and serving for coordinating a high-ohms circuit of the transducer with the input of the measuring device. The attenuator 5 serves for attenuation of input signal. The band-pass filter 6 , made on the "Chebushev scheme" (The Chebyshev filter is an analog filter with a steep slope in the frequency response) of the third order with band of $200-800 \mathrm{kHz}$, selects a portion of the spectrum of a signal, characteristic for cavitation impulses. After the amplification, the signal passes through circuits of the mean-square detector 8 and the amplifier 9 and then moves to indicator. Unit 10 is a power supply of the measuring device and sensor. 


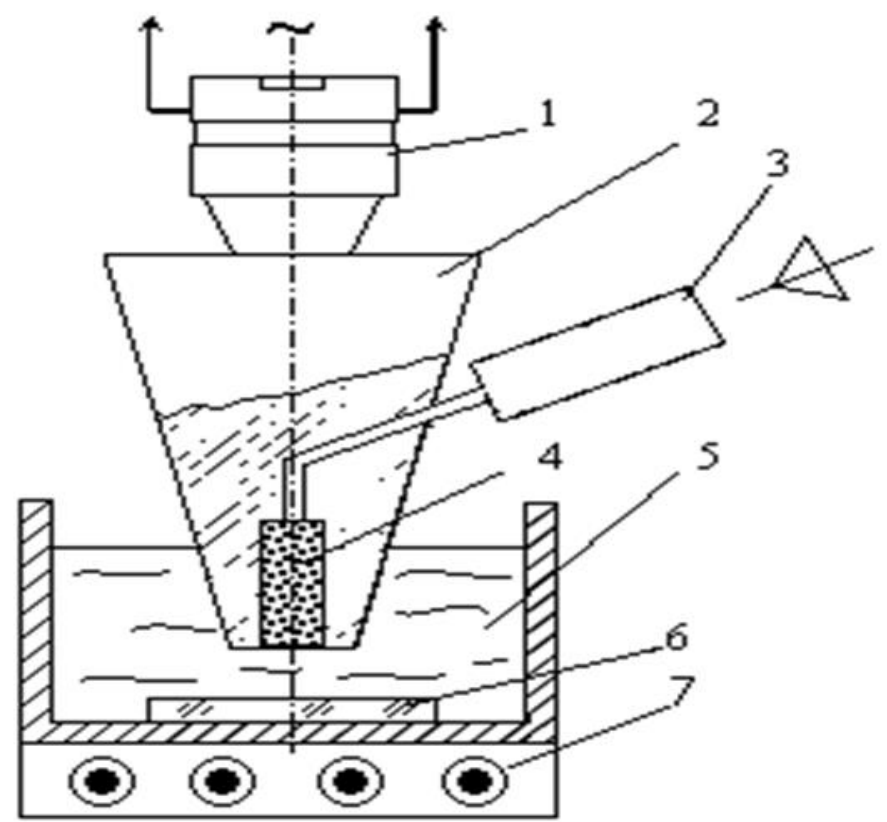

Figure 2. Scheme gas activation in melts (1-US transducer. 2 - US radiator, 3 - tube, 4 - capillary-porous body, 5 - solder, 6 - soldered element, 7 - heater)

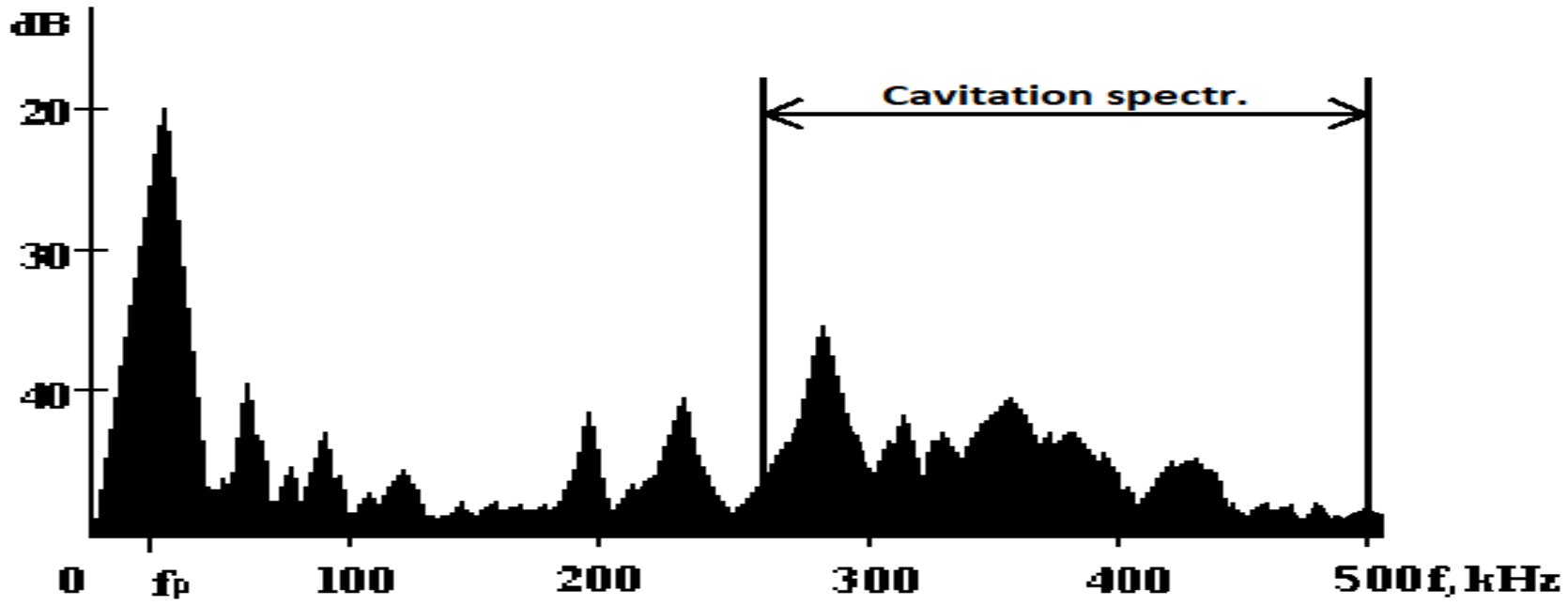

Figure 3. Cavitation noise spectrogram (spectral intensity in decibels of ultrasonic vibrations in solder)

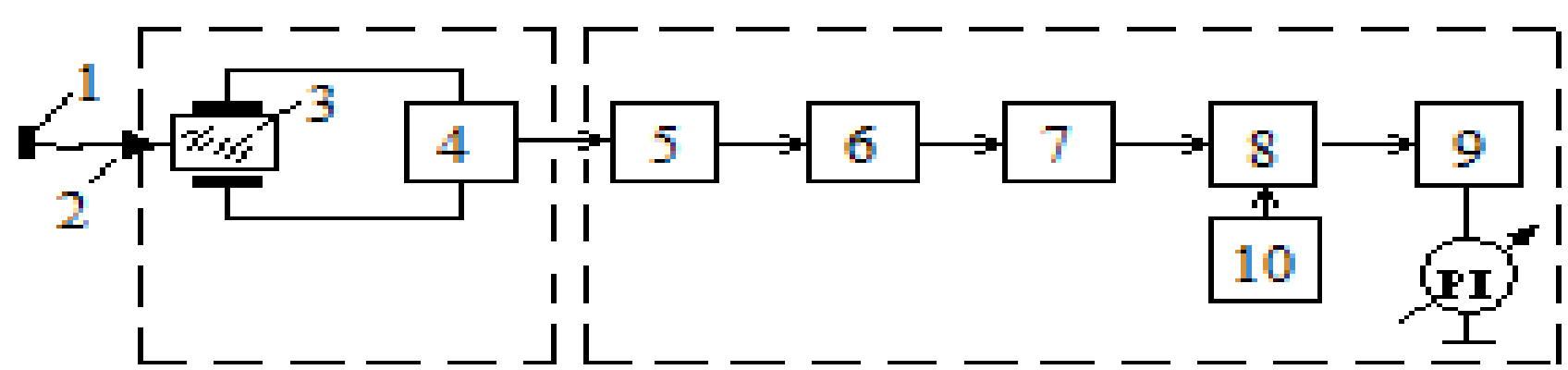

Figure 4. Cavitometer scheme $(1$ - measuring probe, 2 - waveguide, 3 - transducer, 4 - amplifier. 5 attenuator, 6 - band-pass filter, 7 - amplifier, 8 - mean-square detector, 9 - dc amplifier, 10 - unit) 
To the analysis of thermal fields, it is applied mobile thermal camera MobIRM4 with a sensitive element - microbolometer UFPA (160x120 pixels, $35 \mathrm{~mm}$ ), 8-14 microns working in a spectral range. The size of a field of the analysis has made $25^{\circ} \times 19^{\circ}$, focus of $12,6 \mathrm{~mm}$. Sensitivity of the thermal camera $0,1^{\circ} \mathrm{C}$. Measurements were spent on distance of $50 \mathrm{sm}$ from a liquid upper edge. Device adjustment has been made on a reference source of boiling water. For stability of results the measurements were spent in the form of several series after bath warming up in a current of 20 minutes, and then by means of program IR Analyzer V1.7 the thermal fields of distribution of temperatures in the set sites of a bath is received.

The US metallization of glass-ceramic materials was performed in the chamber of the experimental setup (Figure 5.) with a residual rarefaction of 1-10 Pa. For the metallization, a low-melt $\mathrm{Sn}-10 \mathrm{Zn}$ solder and experimentally developed $\mathrm{Pb}-\mathrm{Sn}-\mathrm{Zn}$-In solder on the basis $\mathrm{Sn}$ with the addition of $\mathrm{Zn}$ and in were used [22].

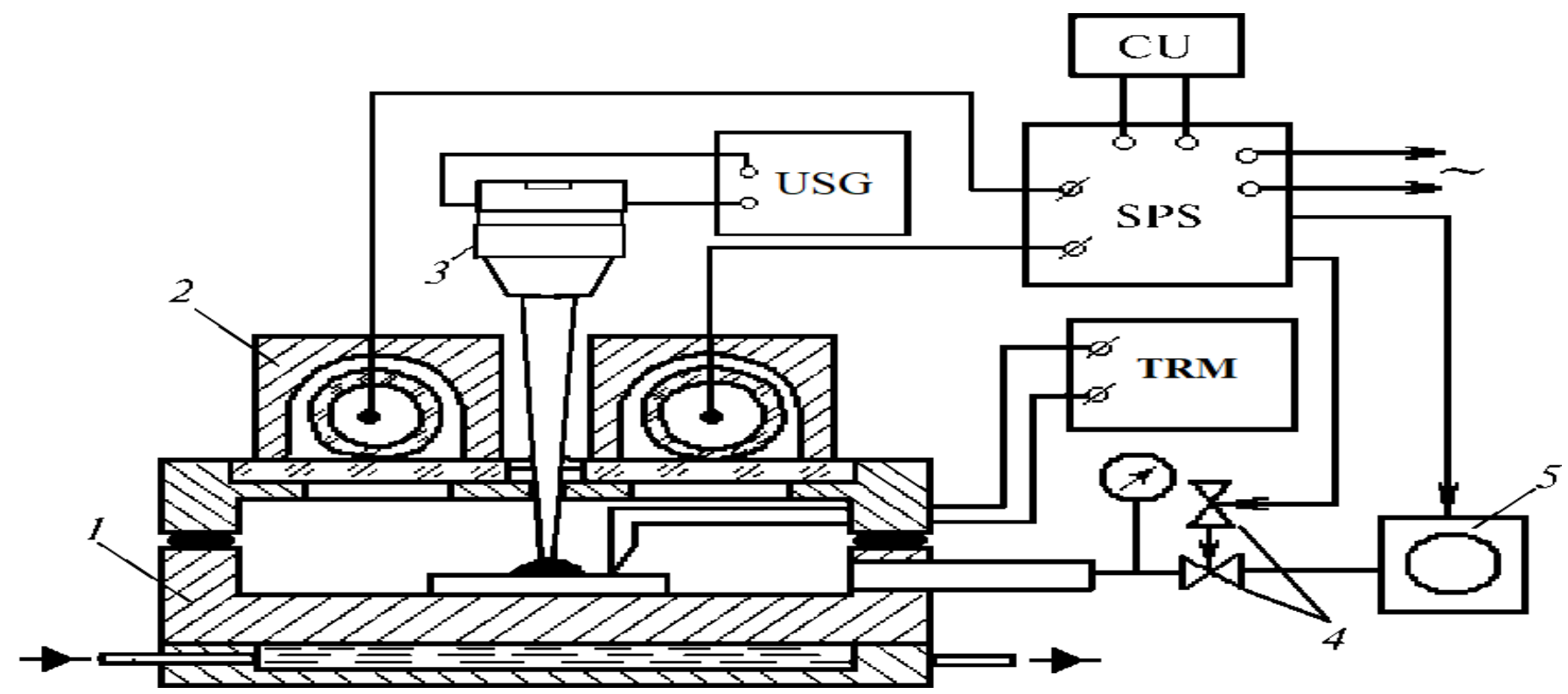

Figure 5. Schema of US and IR radiation activation soldering: 1 - chamber, 2 - IR heater,3-US transducer, 4 - IR valves, 5- compressor

US vibrations with an amplitude of 10-15 mkm and a frequency $22 \mathrm{kHz}$ were introduced into the melt using a radiator in the form of a concentrator of the Fourier type. The melt was heated by IR radiation from two halogen lamps with a power of $1 \mathrm{~kW}$ located in parabolic reflectors. The voltage at the IR lamps was supplied from a stabilized power source (SPS) controlled by a control block (CB). The rate of the IR heating amounted to $10-15^{\circ} \mathrm{C} / \mathrm{sec}$. The temperature of the melt was measured in the working zone using thermocouple and digital measuring device-regulator TRM 201(OVEN).

\section{Results and discussions}

The analysis of thermal fields has shown, that the liquid temperature in US bath as a result of cavitation process raises. The greatest increase (to $10^{\circ} \mathrm{C}$ ) occurs in a zone of the developed cavitation that is connected with warmth allocation at a collapse of cavitation cavities. At gas activation temperature of the liquids near to a nozzle approximately on $0,7^{\circ} \mathrm{C}$ above in comparison with temperature on some distance from it.

Gas saturation of liquids and melts causes increase in cavitation pressure depending on speed of gas and sizes of filter cells. Under the influence of elastic US fluctuations, the formed cavitation's germs pulse in a melt near to a surface of a soldered surface and slam at US pressure $2 \cdot 10^{5} \mathrm{~Pa}$, created on a compression half-cycle. Pressure at which inert gas moved in a melt, depended on viscosity of solder and amplitude US 
fluctuations.

By the help of cavitation indicator, it is established that gas cavities with the sizes 10-50 microns slammed, increasing level of cavitation pressure that activates US processing, including metallization by fusible solders of nonmetallic materials. Local cavitation pressure in a melt at the expense of initiation of a considerable quantity of cavitation germs increased on 20-25\%, and reached a maximum at speed of inert gas of 4-6 $\mathrm{m} / \mathrm{min}$ and the sizes of cells 10 microns (Figure 6.) The big sizes of cavities (over 100 microns) result to formation of a gas cavity which does not slam at levels of US intensity of (2-5) $10^{5} \mathrm{~Pa}$.

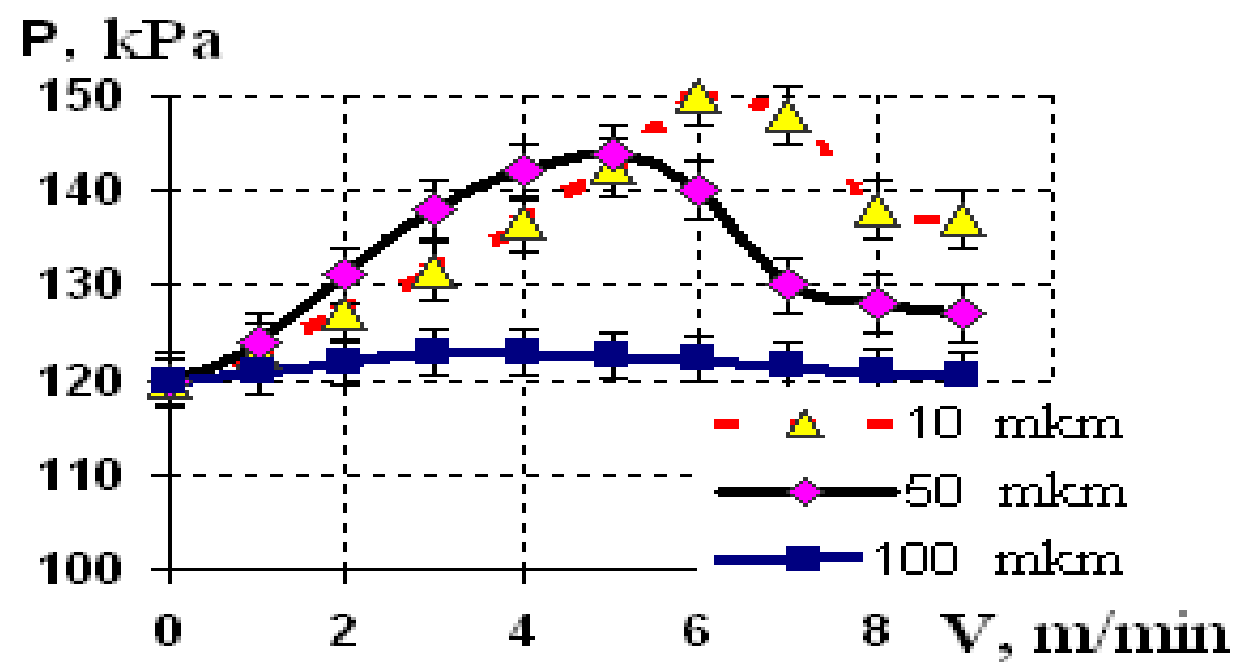

Figure 6. Cavitation pressure vs. speed of gas and sizes of cells

The results of modeling diffusion process showed that the US activation increased the concentration of the diffusing elements of $\mathrm{Zn}$ and $\mathrm{Al}$ in the interface depth by 15-20\% on average, and the combined activation by the US and electric fields increased it by $30-45 \%$. With increase amplitude and frequency of US vibrations, the concentration growth was observed, since the energy quantity adsorbed by melt increased (Figure 7.). The combined activation of melt-soldered material system by US vibrations energy and powerful current pulses additionally increased the heat energy. This allows reaching the soldering temperature at a greater rate, increasing wettability of the surface by solder.

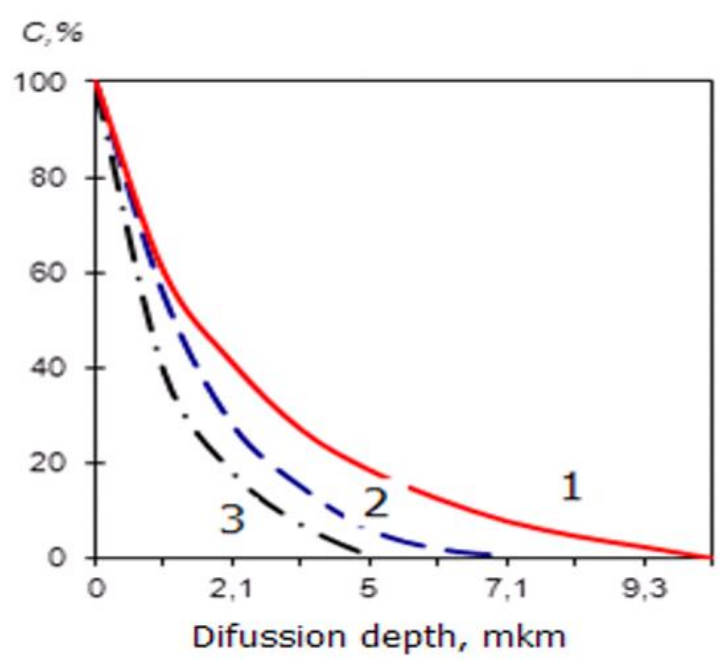

(a)

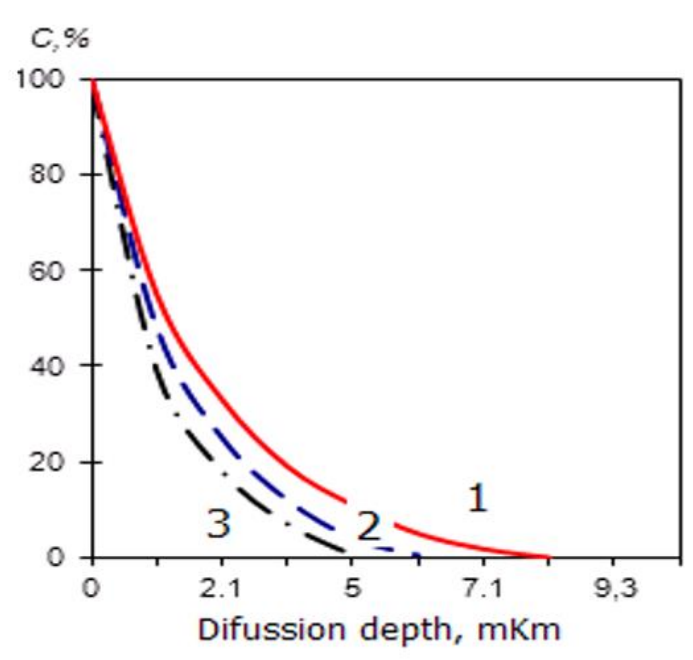

(b)

Figure 7. Effect of US activations on the diffusion concentration: a - amplitude ultrasonic oscillation 20 mkm, b - $10 \mathrm{mkm}$ : 1 - current and US activation, 2 - US activation, 3 - non-activation 
The width of the diffusion zone measured using the scanning electron microscope, in this case, amounted to 4-5 $\mu \mathrm{m}$ for $\mathrm{Sn}-10 \mathrm{Zn}$ and $\mathrm{Sn}-39 \mathrm{~Pb}$ (Figure 8). For the $\mathrm{Sn}-10 \mathrm{Zn}$ solder, the diffusion zone was slightly wider because of the electro mobile $\mathrm{Zn}$ presence in the melt, which migrated to the interface and then deeper in the aluminum alloy thus increasing the width of the diffusion zone. The width of the interface increased to $6-8 \mu \mathrm{m}$ owing to the enhancement of the diffusion interaction and aluminum electro migration into the solder if compared with its size of $1.5 \mu \mathrm{m}$ at the US activation.

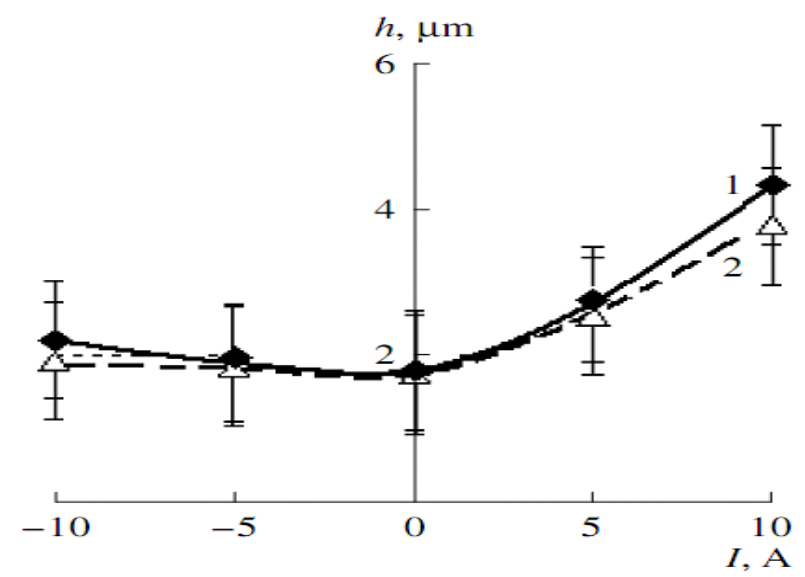

Figure 8. Dependence of the interface width vs. the direction and magnitude of the current pulses in solders: (1) Sn-10Zn, (2) Sn-39Pb.

\section{Conclusions}

Gas saturation of liquids and melts raises level of cavitation pressure upon 20-25\% that allows to intensify US processing, for example, clearings in liquid environments, to increase durability of coupling of metallization with ceramic and glass-ceramic materials, to reduce time of soldering processes, a tinning, metallization, to raise reliability of electronic devices at the expense of reduction of thermal influence.

Practical verification of the combined effect of concentrated energy flows of ultrasonic and electrical current on the processes of contact joints formation of $\mathrm{Al}$ alloys using $\mathrm{Sn}-\mathrm{Pb}$ and $\mathrm{Sn}-\mathrm{Zn}$ solders showed that strength of joints of materials with different chemical composition increases by 1.5-1.8 times due to the intensification of diffusion processes.

Flux-free ultrasonic soldering is non-polluting process and is more economic, as such operations as fluxing and clearing, demanding expenses of time and materials, are excluded. US soldering in some cases is a necessary condition of internal installation and hermetic sealing of the microelectronic equipment. US soldering connect to the help difficult soldering materials: nickel, aluminum, magnesium and titanic alloys, and also nonmetallic materials: ceramics, glass, ferrite. It creates an opportunity of economy of the precious metals rendered on dielectric surfaces of electronic components as metallization.

\section{Disclosure statement}

The author declares that he has no known competing financial interest or personal relationships that could have appeared to influence the work reported in this paper.

\section{References}

[1] Leighton TG, 1994, The Acoustic Bubble. Academic Press, London.

[2] Kolenak R, 2008, Solderability of Metal and Ceramic Materials by Active Solders. Forschungszentrum, Dresden. 
[3] Abramov OV, 1998, High-Intensity Ultrasonics. Theory and Industrial Applications. OPA, Amsterdam.

[4] Naka M, Hafez KM, 2003, Applying of Ultrasonic Waves on Brazing of Alumina to Copper Using Zn-Al Filler Alloy, Journal of Materials Science. 38(16), 3491-3494. DOI:10.1023/A:1025161120349

[5] Lanin VL, 2015, Application of the Concentrated Power Streams in Electronics Industry. Scholar's Press, Saarbrucken.

[6] Ensminger D, Bond LJ, 2012, Ultrasonics: Fundamentals, Technologies, and Applications. CRC Press, Boca Raton.

[7] Subramanian KN, 2007, Lead-Free Electronic Solders. Springer-Science, New York.

[8] Chinnam RK, Fauteux C, Neuenschwander J, Janczak-Rusch J, 2011, Evolution of Microstructure of Sn-Ag-Cu Solder Joints Exposed to Ultrasonic Waves During Solidification, Acta Materialia, 4, 1474-1481. DOI.org/10.1016/j.actamat.

[9] Nagaoka T, Morisada Y, Fukusumi M, Takemoto T, 2009, Strength and Corrosion Resistance of a Solder Joint in Ultrasonic Soldering of Aluminium Using Quasi-Melting Solder. Welding International, 12, 879-885. DOI.org/10.1080/09507110902836960.

[10] Kolenak R, Kostolny I, Drapala J, et al., 2020, Direct Ultrasonic Soldering of AlN Ceramics with Copper Substrate Using Zn-Al-Mg Solder. Metals. 10(2), 1-18. https://doi.org/10.3390 /met10020160.

[11] Dušek K, Plaček M, Čepek M, et al., 2017, Ultrasonic Soldering, Mechanical Properties of Solder Joints. Proc. of 40th International Spring Seminar on Electronics Technology (ISSE). 10-14 May 2017. Sofia, Bulgaria, 1-4.

[12] 2008, Technical Data Sheet BALVER ZINN Solder. https://www.yumpu.com > document > view > tech.

[13] Belyakov SA, 2008, Ultrasonic Modification of Sn-Ag-Cu Alloy Microstructure. Proc. of $9^{\text {th }}$ Inter. Workshop and Tutorials, EDM'2008. Erlagol, 58-59.

[14] Faridi H, Devletian J, Le H, 2000, A New Look at Flux-Free Ultrasonic Soldering. Welding Journal. 9, 41-45.

[15] Pericleous K, Bojarevics V, Djambazov G, et al., 2019, Contactless Ultrasonic Cavitation in Alloy Melts, Materials. 12, 1-13. https://doi.org/10.3390/ma12213610

[16] Agranat BA, Gudovich AP, Nezhenko LB, 1986, Ultrasound in Powder Metallurgy. Metallurgy, Moscow.

[17] Shilyaev AS, 1992, Ultrasonic Treatment of Melts in the Production and Restoration of Machine Parts. Science and Technique, Minsk.

[18] Lanin VL, 2008, Activation of Soldered Connections in the Process of Formation Using the Energy of Ultrasonic and Electric Fields, Surface Engineering and Applied Electrochemistry. 3, 234-239. https://doi: 10.3103/S1068375508030113.

[19] Lanin VL, 2013, Increase Activity Cavitations in Liquids and Melts at Ultrasonics Processing, Open Journal of Acoustics. 3A, 13-15. https:// doi:10.4236/oja.2013.33A003

[20] Ilyichev VI, Koretz VL, Melnicov NP, 1989, Spectral Characteristics of Acoustic Cavitation., Ultrasonics. 27, 357-361.

[21] Lanin VL, 2003, Cavitation Intensity Investigation at Ultrasonic Soldering. Proc. of Ultrasonics Int. Conf., 1-3 July 2003, Granada (Spain), 154-156.

[22] Lanin VL, 2010, Ultrasonic Soldering in Electronics: New Opportunities, Solid State Electrochemistry by T. G. Willard. 2010. Nova Science Publishers, New York: 201-211. 